\title{
Analytical relations describing piezooptic effect in tetragonal crystals
}

\author{
${ }^{1,2}$ Mytsyk B., ${ }^{2}$ Demyanyshyn N. and ${ }^{2}$ Kost' Ya. \\ ${ }^{1}$ Institute of Physical Optics, 23 Dragomanov St., 79005 Lviv, Ukraine \\ ${ }^{2}$ Karpenko Physics and Mechanics Institute, 5 Naukova St., 79601 Lviv, Ukraine
}

Received: 12.03 .2013

\begin{abstract}
We have derived analytical relations necessary for interpreting piezooptic effect and calculation of all piezooptic coefficients on the basis of interferometric technique, which take a micro-wedge shape of samples into consideration. The analysis is presented for the tetragonal crystals of point symmetry classes $4, \overline{4}$ and $4 / \mathrm{m}$. The relations valid for the remaining symmetry classes $422,4 \mathrm{~mm}, \overline{4} 2 \mathrm{~m}$ and $4 / \mathrm{mmm}$ represent simple particular cases of the general relations.
\end{abstract}

Keywords: piezooptic effect, optical indicatrix, mechanical stress, elastic strain, interferometric techniques.

PACS: $78.20 . \mathrm{Hp}$

UDC: 535.551

\section{Introduction}

Experimental studies of piezooptic effect (POE) in crystalline materials do not involve remarkable problems whenever one deals with the crystals belonging to the cubic or orthorhombic systems and higher-symmetry groups of the tetragonal and hexagonal systems $(4 \mathrm{~mm}, 422, \overline{4} 2 \mathrm{~m}, 4 / \mathrm{mmm}$, $6 \mathrm{~mm}, 622, \overline{6} \mathrm{~m} 2$, and $6 / \mathrm{mmm}$ ) [1-8]. The matter is that the tensors describing POE for these symmetry groups include only so-called 'principal' components ( $\pi_{i m}$, with $i, m=1,2,3$ and the indices $i, m$ corresponding to the directions of light polarization and uniaxial mechanical stress, respectively) and 'diagonal' components $\pi_{44}, \pi_{55}$ and $\pi_{66}$. On the other hand, there can happen more complicated situation when the POE tensor contains both 'non-principal' and 'non-diagonal' components such as $\pi_{14}, \pi_{41}, \pi_{45}, \pi_{16}$ etc. Among the well-known crystalline materials, the examples are $\mathrm{LiNbO}_{3}$ and $\mathrm{LiNbO}_{3}: \mathrm{MgO}$ (the point symmetry class $3 \mathrm{~m}$ ), and $\mathrm{La}_{3} \mathrm{Ga}_{5} \mathrm{SiO}_{11}$ (the class 32) [9, 10]. Nonetheless, the $\pi_{14}$ coefficients have been determined for $\mathrm{LiNbO}_{3}$ and $\alpha-\mathrm{BaB}_{2} \mathrm{O}_{4}$ (the symmetry class $\overline{3} \mathrm{~m}$ ) using a so-called torsion method [11, 12].

Following from the matrices of piezooptic coefficients (POCs), the tetragonal crystals may be divided into two subgroups:

(1) the first one embraces the symmetry classes $4, \overline{4}$ and 4/m; they are characterised by complicated POC matrices with ten independent coefficients, including five non-principal ones (a so-called 'rotational' coefficient $\pi_{61}$, a 'shifting' coefficient $\pi_{16}$, and 'rotational-shifting' coefficients $\pi_{44}, \pi_{45}$ and $\left.\pi_{66}\right)$

(2) the second subgroup embraces the groups $422,4 \mathrm{~mm}, \overline{4} 2 \mathrm{~m}$ and $4 / \mathrm{mmm}$, with seven independent coefficients; only two of these, $\pi_{44}$ and $\pi_{66}$, are non-principal.

The crystals belonging to the first, lower-symmetry subgroup of the tetragonal system (the 
classes $4, \overline{4}$ and $4 / \mathrm{m}$ ) reveal the same form of the POE tensor $[8,13]$. We are to stress that, up to date, no reliable experimental data have been reported for their POCs. This is due to lack of relevant analytical relations, which are necessary for interpreting the experimental results and calculating the non-principal POCs on this basis. The aim of the present work is just to solve this problem. Below we will describe the POE for the first tetragonaal subgroup only, since the analytical relations for the second one represent particular cases of the more complicated relations for the first subgroup.

\section{Opening remarks and formulation of the problem}

In order to study the POE for the crystals belonging to the first tetragonal subgroup, it is necessary to prepare five samples with different crystallographic orientations shown schematically in Fig. 1. At the same time, samples of only three different orientations are needed for the crystals of the second subgroup (see Fig. 1a, b, and c).

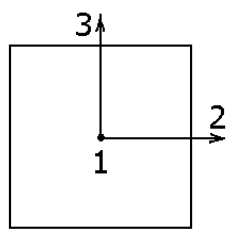

a)

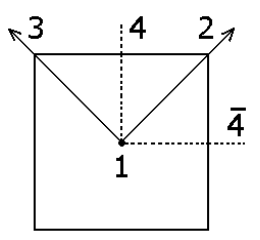

b)

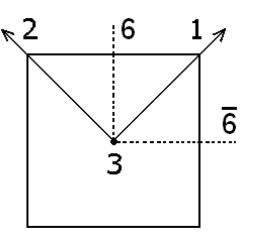

c)

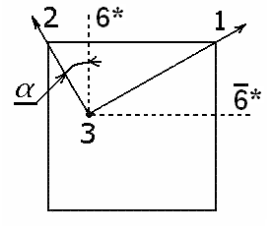

d)

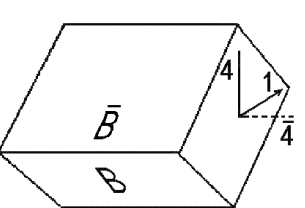

e)

Fig. 1. Schemes of sample orientations for studying the POE in tetragonal crystals: (a) direct crystallographic cut sample, (b) $X / 45^{\circ}$-cut, (c) $Z / 45^{\circ}$-cut, (d) $Z / 22.5^{\circ}$-cut $\left(\alpha=22.5^{\circ}\right.$ ), and (e) $B$-cut (see the text).

Let us remind that the principal POCs $(i, m=1,2,3)$ can be obtained on the basis of theoretical relations derived in the works $[9,10]$. The most common experimental technique is based on interferometric measurements and additional consideration of experimental errors that appear due to micro-wedge shape of a sample:

$$
\pi_{i m}=-\frac{\lambda}{2 n_{i}^{3}}\left(\frac{1}{\sigma_{i m}^{o}}+\frac{1}{\sigma_{i m}^{\prime o}}\right)+\frac{2 S_{k m}}{n_{i}^{3}}\left(n_{i}-1\right) .
$$

Here $\lambda$ is the light wavelength in vacuum, $n_{i}$ the refractive index of crystal, $S_{k m}$ the components of elastic compliance tensor, and $\sigma_{i m}^{o}$ and $\sigma_{i m}^{\prime o}=\sigma_{i m} d_{k}$ denote the operating stresses defined with respect to a preceding cycle of measurements (for any details see [9]). In the last formulae, the parameter $\sigma_{i m}$ means the stress inducing a half-wave phase retardation, $d_{k}$ the sample thickness along the direction of light wave vector $k$, and $\sigma_{i m}^{\prime o}$ the operating stress for the wedgelike sample rotated by $180^{\circ}$ around the direction of light beam.

For completing the POC matrix of the tetragonal crystals, one should rely on the analytical relations for the non-principal POCs $\pi_{44}, \pi_{66}, \pi_{61}, \pi_{16}$, and $\pi_{45}$, which are analogues of the relations derived earlier in the study [14]. Unfortunately, the theoretical relations suggested in the work [14] are characterised by a number of disadvantages. Below we will itemise them and outline the possible ways out.

1. The relations mentioned above facilitate obtaining only the two coefficients, $\pi_{44}$ and $\pi_{66}$. Moreover, these relations are written in a form that excludes any possibility for consideration of a 
wedge-like shape of real experimental samples, when determining the half-wave stresses. However, it is known that a neglect of even very small wedges (a case which we refer to as a 'microwedge') can lead to notable errors while interpreting the experimental results.

Let us notice that the $\pi_{66}$ coefficient appears in the theoretical relations with its sign undefined.

It means that one should somehow distinguish between the directions 6 and $\overline{6}$ (see Fig. 1c). This is a difficult problem. Indeed, usually the positive directions of the principal axes $X_{1}, X_{2}$ and $X_{3}$ (or simply 1,2 and 3) of the optical ellipsoid are determined and the directions 4, 5 and 6 are distinguished respectively from $\overline{4}, \overline{5}$ and $\overline{6}$ using a piezoelectric effect [13, 14]. However, since the crystals of the point group $4 / \mathrm{m}$ reveal no piezoelectric effect, it is necessary to choose some other criteria to define the directions 6 and $\overline{6}$ and then determine the sign of the coefficient $\pi_{66}$. The simplest solution is as follows. The magnitude and the sign of the POC $\pi_{66}$ can be found using a relation which does not contain the indefinite sign, i.e. corresponds to some other experimental condition [14]). Then the relation that involves the uncertainty " $\pm \pi_{66}$ " can be used only to determine more accurately the magnitude of the $\pi_{66}$ coefficient. This becomes possible since the latter relation contains the Poisson contribution of a very simple from and, therefore, the relevant error contributes very little to the total experimental error of the $\pi_{66}$ coefficient.

2. The relations used for determination of the coefficients $\pi_{16}, \pi_{61}$ and $\pi_{45}$ include cumbersome combinations of these POCs. Thus it is impossible to determine these coefficients separately.

3. The experimental conditions enabling determination of the sums of POCs $\pi_{11}+\pi_{12}$ have not yet been considered. Moreover, it would be very useful to compare these sums found experimentally with the same sums calculated from the involved coefficients obtained in independent and separate experiments.

It is obvious that any reliable studies of the POE for the tetragonal crystals of the first subgroup require theoretical relations enabling separate determination of the coefficients $\pi_{16}, \pi_{61}$ and $\pi_{45}$. These relations should be written for the two right-handed coordinate systems, for which the directions 6 and $\overline{6}, 6^{*}$ and $\overline{6}^{*}$, and 4 and $\overline{4}$ are interchanged (see Fig. 1c, d, e). The latter can, in principle, help finding out the conditions for unambiguous determination of these POCs. So-called 'symmetric conditions' of piezooptic experiments should also be considered (see Table 1), with further analysis of the relations obtained. In case if the relations turn out to be different for these experimental conditions, one should formulate the relevant recommendations for unambiguous determination of the non-principal POCs.

Table 1. Analytical relations for determination of POCs in the crystals of symmetry classes $4, \overline{4}$ and 4/m using half-wave stress technique, which accounting for micro-wedge shape of samples

\begin{tabular}{|l|c|c|}
\hline $\begin{array}{c}\text { Experimen- } \\
\text { tal condi- } \\
\text { tions }\end{array}$ & Relations \\
\hline \multicolumn{3}{|c|}{ Sample of $X / 45^{\circ}$-cut } \\
\hline \begin{tabular}{l|l}
$m=4(\overline{4})$ \\
$k=\overline{4}(4)$ \\
$i=4(\overline{4})$
\end{tabular} & $\pi_{11}+\pi_{13}+\pi_{31}+\pi_{33}+2 \pi_{44}=-\frac{2 \lambda}{n_{4}^{3}}\left(\frac{1}{\sigma_{44(\overline{44})}^{o}}+\frac{1}{\sigma_{44(\overline{44})}^{o l}}\right)$ & $\mathrm{T} .1$ \\
\hline
\end{tabular}

Ukr. J. Phys. Opt. 2013, Volume 14, Issue 3 
Mytsyk B. et al

\begin{tabular}{|c|c|c|}
\hline $\begin{array}{l}\text { Experimen- } \\
\text { tal condi- }\end{array}$ & \multicolumn{2}{|l|}{ Relations } \\
\hline $\begin{array}{l}m=1 \\
k=4(\overline{4}) \\
i=1\end{array}$ & $\pi_{11}=-\frac{\lambda}{2 n_{1}^{3}}\left(\frac{1}{\sigma_{11}^{o}}+\frac{1}{\sigma_{11}^{\prime o}}\right)_{k=4(\overline{4})}+\left(S_{12}+S_{13}\right) \frac{n_{1}-1}{n_{1}^{3}}$ & Т.2 \\
\hline $\begin{array}{l}m=4(\overline{4}) \\
k=\overline{4}(4) \\
i=1\end{array}$ & $\pi_{12}+\pi_{13}=-\frac{\lambda}{n_{1}^{3}}\left(\frac{1}{\sigma_{14(1 \overline{4})}^{o}}+\frac{1}{\sigma_{14(1 \overline{4})}^{o}}\right)+\left(S_{11}+2 S_{13}+S_{33}-S_{44}\right) \frac{n_{1}-1}{n_{1}^{3}}$ & Т.3 \\
\hline $\begin{array}{l}m=1 \\
k=\overline{4}(4) \\
i=4(\overline{4})\end{array}$ & $\pi_{12}+\pi_{31}=-\frac{\lambda}{n_{4}^{3}}\left(\frac{1}{\sigma_{41(\overline{4} 1)}^{o}}+\frac{1}{\sigma_{41(\overline{4} 1)}^{o}}\right)+2\left(S_{12}+S_{13}\right) \frac{n_{4}-1}{n_{4}^{3}}$ & T.4 \\
\hline $\begin{array}{l}m=4(\overline{4}) \\
k=1 \\
i=2\end{array}$ & $\pi_{11}+\pi_{13}=-\frac{\lambda}{n_{1}^{3}}\left(\frac{1}{\sigma_{24(2 \overline{4})}^{o}}+\frac{1}{\sigma_{24(2 \overline{4})}^{\prime o}}\right)+2\left(S_{12}+S_{13}\right) \frac{n_{1}-1}{n_{1}^{3}}$ & T.5 \\
\hline $\begin{array}{l}m=4(\overline{4}) \\
k=1 \\
i=3\end{array}$ & $\pi_{31}+\pi_{33}=-\frac{\lambda}{n_{3}^{3}}\left(\frac{1}{\sigma_{34(3 \overline{4})}^{o}}+\frac{1}{\sigma_{34(3 \overline{4})}^{\prime o}}\right)+2\left(S_{12}+S_{13}\right) \frac{n_{3}-1}{n_{3}^{3}}$ & T.6 \\
\hline \multicolumn{3}{|c|}{ Sample of $Z / 45^{\circ}$-cut } \\
\hline $\begin{array}{l}m=3 \\
k=6(\overline{6}) \\
i=3\end{array}$ & $\pi_{33}=-\frac{\lambda}{2 n_{3}^{3}}\left(\frac{1}{\sigma_{33}^{o}}+\frac{1}{\sigma_{33}^{\prime o}}\right)_{k=6(\overline{6})}+2 S_{13} \frac{n_{3}-1}{n_{3}^{3}}$ & Т.7 \\
\hline $\begin{array}{l}m=3 \\
k=\overline{6}(6) \\
i=6(\overline{6})\end{array}$ & $\pi_{13}=-\frac{\lambda}{2 n_{1}^{3}}\left(\frac{1}{\sigma_{63(\overline{6} 3)}^{o}}+\frac{1}{\sigma_{63(\overline{6} 3)}^{\prime o}}\right)+2 S_{13} \frac{n_{1}-1}{n_{1}^{3}}$ & Т.8 \\
\hline $\begin{array}{l}m=6(\overline{6}) \\
k=\overline{6}(6) \\
i=6(\overline{6})\end{array}$ & $\pi_{11}+\pi_{12}+\pi_{66}=-\frac{\lambda}{n_{1}^{3}}\left(\frac{1}{\sigma_{66(\overline{66})}^{o}}+\frac{1}{\sigma_{66(\overline{66})}^{o}}\right)+\left(2 S_{11}+2 S_{12}-S_{66}\right) \frac{n_{1}-1}{n_{1}^{3}}$ & T.9 \\
\hline $\begin{array}{l}m=6(\overline{6}) \\
k=\overline{6}(6) \\
i=3\end{array}$ & $\pi_{31}=-\frac{\lambda}{2 n_{3}^{3}}\left(\frac{1}{\sigma_{36(3 \overline{6})}^{o}}+\frac{1}{\sigma_{36(3 \overline{6})}^{\prime o}}\right)+\frac{1}{2}\left(2 S_{11}+2 S_{12}-S_{66}\right) \frac{n_{3}-1}{n_{3}^{3}}$ & T.10 \\
\hline \multicolumn{3}{|c|}{ Sample of $Z / 45^{\circ}$-cut } \\
\hline $\begin{array}{l}m=6 \\
k=3 \\
i=6(\overline{6})\end{array}$ & $\begin{array}{l}\pi_{66}=-\frac{\lambda}{2 n_{1}^{3}}\left(\frac{1}{\sigma_{66(\overline{6} \overline{6})}^{o}}+\frac{1}{\sigma_{66(\overline{6} \bar{b})}^{\prime O}}-\frac{1}{\sigma_{\overline{6} 6(6 \overline{6})}^{o}}-\frac{1}{\sigma^{\prime \prime} \overline{6}(6 \overline{\bar{b}})}\right) \\
\text { for the indices in brackets one has } m=\overline{6}, k=3 \text {, and } i=\overline{6}(6)\end{array}$ & T.11 \\
\hline $\begin{array}{l}m=6 \\
k=3 \\
i=6(\overline{6})\end{array}$ & $\begin{array}{l}\pi_{11}+\pi_{12}=-\frac{\lambda}{2 n_{1}^{3}}\left(\frac{1}{\sigma_{66(\overline{66})}^{o}}+\frac{1}{\sigma_{66(\overline{66})}^{\prime o}}+\frac{1}{\sigma_{\overline{6} 6(6 \overline{6})}^{o}}+\frac{1}{\sigma^{\prime} \frac{0}{66(6 \overline{6})}}\right)+4 S_{13} \frac{n_{1}-1}{n_{1}^{3}} \\
\text { for the indices in brackets one has } m=\overline{6}, k=3 \text {, and } i=\overline{6}\end{array}$ & T.12 \\
\hline \multicolumn{3}{|c|}{ Sample of $Z / 22.5^{\circ}$-cut } \\
\hline $\begin{array}{l}m=3 \\
k=6^{*}\left(\overline{6}^{*}\right) \\
i=3\end{array}$ & $\pi_{33}=-\frac{\lambda}{2 n_{3}^{3}}\left(\frac{1}{\sigma_{33}^{o}}+\frac{1}{\sigma_{33}^{o}}\right)_{k=6^{*}\left(\overline{6^{*}}\right)}+2 S_{13} \frac{n_{3}-1}{n_{3}^{3}}$ & T.13 \\
\hline $\begin{array}{l}m=3 \\
k=\overline{6}^{*}\left(6^{*}\right) \\
i=6^{*}\left(\overline{6}^{*}\right)\end{array}$ & $\pi_{13}=-\frac{\lambda}{2 n_{1}^{3}}\left(\frac{1}{\sigma_{6^{*} 3\left(\overline{6}^{*} 3\right)}^{o}}+\frac{1}{\sigma_{6^{*} 3\left(\overline{6}^{*} 3\right)}^{o}}\right)+2 S_{13} \frac{n_{1}-1}{n_{1}^{3}}$ & T.14 \\
\hline
\end{tabular}




\begin{tabular}{|c|c|c|}
\hline $\begin{array}{l}\text { Experimen- } \\
\text { tal condi- } \\
\text { tions }\end{array}$ & Relations & \\
\hline $\begin{array}{l}m=6^{*}\left(\overline{6}^{*}\right) \\
k=\overline{6}^{*}\left(6^{*}\right) \\
i=6^{*}\left(\overline{6}^{*}\right)\end{array}$ & $\begin{array}{l}\pi_{11}\left(1+\tan ^{4} \alpha\right)+2\left(\pi_{12}+\pi_{66}\right) \tan ^{2} \alpha+\left(\pi_{16}+2 \pi_{61}\right)\left(\tan ^{3} \alpha-\tan \alpha\right) \\
=-\frac{\lambda}{2 n_{1}^{3} \cos ^{4} \alpha}\left(\frac{1}{\sigma_{6^{*} 6^{*}\left(6^{*} 6^{*}\right)}^{o}}+\frac{1}{\sigma_{6^{*} 6^{*}\left(6^{*} \sigma^{*}\right)}^{\prime}}\right)+2\left[\left(2 S_{11}-S_{66}\right) \tan ^{2} \alpha\right. \\
\left.+S_{12}\left(1+\tan ^{4} \alpha\right)-2 S_{16}\left(\tan ^{3} \alpha-\tan \alpha\right)\right]\left(n_{1}-1\right) / n_{1}^{3}\end{array}$ & T.15 \\
\hline $\begin{array}{l}m=6^{*}\left(\overline{6}^{*}\right) \\
k=\overline{6}^{*}\left(6^{*}\right) \\
i=3\end{array}$ & $\begin{array}{l}\pi_{31}=-\frac{\lambda}{2 n_{3}^{3}}\left(\frac{1}{\sigma_{36^{*}\left(36^{*}\right)}^{o}}+\frac{1}{\sigma_{36^{*}\left(3 \overline{6}^{*}\right)}^{\prime o}}\right)+2 \cos ^{4} \alpha \cdot\left[\left(2 S_{11}-S_{66}\right) \tan ^{2} \alpha\right. \\
\left.+S_{12}\left(1+\tan ^{4} \alpha\right)-2 S_{16}\left(\tan ^{3} \alpha-\tan \alpha\right)\right]\left(n_{3}-1\right) / n_{3}^{3}\end{array}$ & T.16 \\
\hline $\begin{array}{l}m=6^{*}\left(\overline{6}^{*}\right) \\
k=3 \\
i=6^{*}\left(\overline{6}^{*}\right)\end{array}$ & $\begin{array}{l}\pi_{11}\left(1+\tan ^{4} \alpha\right)+2\left(\pi_{12}+\pi_{66}\right) \tan ^{2} \alpha+\left(\pi_{16}+2 \pi_{61}\right)\left(\tan ^{3} \alpha-\tan \alpha\right) \\
=-\frac{\lambda}{2 n_{1}^{3} \cos ^{4} \alpha}\left(\frac{1}{\sigma_{6^{*} 6^{*}\left(\bar{\sigma}^{*} \overline{6}^{*}\right)}^{o}}+\frac{1}{\sigma_{6^{*} 6^{*}\left(\overline{6}^{*} \overline{6}^{*}\right)}^{0}}\right)+\frac{2 S_{13}}{\cos ^{4} \alpha} \cdot \frac{n_{1}-1}{n_{1}^{3}}\end{array}$ & T.17 \\
\hline $\begin{array}{l}m=6^{*}\left(\overline{6}^{*}\right) \\
k=3 \\
i=\overline{6}^{*}\left(6^{*}\right)\end{array}$ & $\begin{array}{l}\pi_{12}\left(1+\tan ^{4} \alpha\right)+2\left(\pi_{11}-\pi_{66}\right) \tan ^{2} \alpha-\left(\pi_{16}+2 \pi_{61}\right)\left(\tan ^{3} \alpha-\tan \alpha\right) \\
=-\frac{\lambda}{2 n_{1}^{3} \cos ^{4} \alpha}\left(\frac{1}{\sigma_{6^{*}}^{o} 6^{*}\left(6^{*} \overline{6}^{*}\right)}+\frac{1}{\sigma^{\prime o}{ }^{*} 6^{*}\left(6^{*} \sigma^{*}\right)}\right)+\frac{2 S_{13}}{\cos ^{4} \alpha} \cdot \frac{n_{1}-1}{n_{1}^{3}}\end{array}$ & Т.18 \\
\hline $\begin{array}{l}m=6^{*} \\
k=3 \\
i=6^{*}\left(\overline{6}^{*}\right)\end{array}$ & $\begin{array}{l}2\left(\pi_{16}+2 \pi_{61}\right)\left(\tan ^{3} \alpha-\tan \alpha\right)=-\frac{\lambda}{2 n_{1}^{3} \cos ^{4} \alpha} \\
\times\left(\frac{1}{\sigma_{6^{*} 6^{*}}^{o}}+\frac{1}{\sigma_{6^{*} 6^{*}}^{\prime o}}-\frac{1}{\sigma_{6^{*} 6^{*}}^{o}}-\frac{1}{\sigma_{\frac{6}{6} 6^{*}}^{o}}\right)-\left(\pi_{11}-\pi_{12}\right)\left(1-\tan ^{2} \alpha\right)^{2}-4 \pi_{66} \tan ^{2} \alpha\end{array}$ & T.19 \\
\hline & Sample of $Z / 22.5^{\circ}$-cut & \\
\hline $\begin{array}{l}m=\overline{6}^{*} \\
k=3 \\
i=\overline{6}^{*}\left(6^{*}\right)\end{array}$ & $\begin{array}{l}2\left(\pi_{16}+2 \pi_{61}\right)\left(\tan ^{3} \alpha-\tan \alpha\right) \\
=-\frac{\lambda}{2 n_{1}^{3} \cos ^{4} \alpha}\left(\frac{1}{\sigma_{\overline{6}^{*} \overline{6}^{*}}^{o}}+\frac{1}{\sigma_{\overline{6}^{*} \overline{6}^{*}}^{o}}-\frac{1}{\sigma_{6^{*} \overline{6}^{*}}^{o}}-\frac{1}{\sigma_{6^{*} \overline{6}^{*}}^{o}}\right) \\
-\left(\pi_{11}-\pi_{12}\right)\left(1-\tan ^{2} \alpha\right)^{2}-4 \pi_{66} \tan ^{2} \alpha\end{array}$ & Т.20 \\
\hline $\begin{array}{l}m=6^{*} \\
k=3 \\
i=6^{*}\left(\overline{6}^{*}\right)\end{array}$ & $\pi_{11}+\pi_{12}=-\frac{\lambda}{2 n_{1}^{3}}\left(\frac{1}{\sigma_{6^{*} 6^{*}}^{o}}+\frac{1}{\sigma_{6^{*} 6^{*}}^{\prime o}}+\frac{1}{\sigma_{6^{*} 6^{*}}^{o}}+\frac{1}{\sigma_{6^{*} 6^{*}}^{o}}\right)+4 S_{13} \frac{n_{1}-1}{n_{1}^{3}}$ & Т. 21 \\
\hline $\begin{array}{l}m=\overline{6}^{*} \\
k=3 \\
i=\overline{6}^{*}\left(6^{*}\right)\end{array}$ & $\pi_{11}+\pi_{12}=-\frac{\lambda}{2 n_{1}^{3}}\left(\frac{1}{\sigma_{\overline{6}^{*} \overline{6}^{*}}^{o}}+\frac{1}{\sigma_{\overline{6}^{*} \overline{6}^{*}}^{10}}+\frac{1}{\sigma_{6^{*} \sigma^{*}}^{o}}+\frac{1}{\sigma_{6^{*} \sigma^{*}}^{10}}\right)+4 S_{13} \frac{n_{1}-1}{n_{1}^{3}}$ & T.22 \\
\hline & Sample of $B$-cut & \\
\hline $\begin{array}{l}m=\perp B(\bar{B}) \\
k=\overline{4} \\
i=1\end{array}$ & $\begin{array}{l}2 \pi_{11}+\pi_{12}+\pi_{13} \pm \sqrt{2} \pi_{16}=-\frac{2 \lambda}{n_{1}^{3}}\left(\frac{1}{\sigma_{1 B(1 \bar{B})}^{o}}+\frac{1}{\sigma_{1 B(1 \bar{B})}^{\prime o}}\right) \\
+2\left(S_{11}+S_{33}+2 S_{12}+4 S_{13}-S_{44} \mp \sqrt{2} S_{16}\right) \frac{n_{1}-1}{n_{1}^{3}}\end{array}$ & Т. 23 \\
\hline
\end{tabular}




\begin{tabular}{|l|l|l|}
\hline $\begin{array}{l}\text { Experimen- } \\
\text { tal condi- } \\
\text { tions }\end{array}$ & \multicolumn{2}{|c|}{ Relations } \\
\hline $\begin{array}{l}m=\perp B(\bar{B}) \\
k=\overline{4} \\
i=1\end{array}$ & $\pi_{16}=-\frac{\lambda}{\sqrt{2} n_{1}^{3}}\left(\frac{1}{\sigma_{1 B}^{o}}+\frac{1}{\sigma_{1 B}^{\prime o}}-\frac{1}{\sigma_{1 \bar{B}}^{o}}-\frac{1}{\sigma_{1 \bar{B}}^{\prime o}}\right)-S_{16} \frac{n_{1}-1}{n_{1}^{3}}$ & T.24 \\
\hline $\begin{array}{l}m=\perp B(\bar{B}) \\
k=\overline{4} \\
i=4\end{array}$ & $\pi_{11}+\pi_{13}+\pi_{33}+3 \pi_{31}+2\left(\pi_{12}+\pi_{44}\right) \pm \sqrt{2}\left(2 \pi_{45}-\pi_{16}\right)$ & \\
\hline $\begin{array}{l}m=\perp B(\bar{B}) \\
k=\overline{4} \\
i=4\end{array}$ & $=-\frac{4 \lambda}{n_{4}^{3}}\left(\frac{1}{\sigma_{4 B(4 \bar{B}}^{o}}+\frac{1}{\sigma_{4 B(4 \bar{B})}^{\prime o}}\right)+2\left(S_{11}+S_{33}+2 S_{12}+4 S_{13}-S_{44} \mp \sqrt{2} S_{16}\right) \frac{n_{4}-1}{n_{4}^{3}}$ & T.25 \\
\hline
\end{tabular}

Footnotes: (1) in cases when the operating stresses appearing in a formula are indicated by indices in brackets (e.g., $\sigma_{41(\overline{4})}^{o}$ ), one should put $\sigma_{41}^{o}$ into the formula for the direct experimental conditions and $\sigma_{\overline{4} 1}^{o}$ for the symmetric conditions; (2) the upper and lower signs of the terms $\pi_{16}, 2 \pi_{45}-\pi_{16}$ and $S_{16}$ appearing in Eqs. (T.23) and (T.25) for the $B$-cut sample correspond to the conditions $m \perp B$ and $m \perp \bar{B}$, respectively.

\section{Experimental manifestations of POE for a $X / 45^{\circ}$-cut sample}

Consider the relation used for determination of $\pi_{44}$ basing on the measurements of optical path change $\delta \Delta_{k}$ at $i=4, k=\overline{4}$ and $m=4[14]$ :

$$
\delta \Delta_{\overline{4}}=-\frac{1}{8}\left(\pi_{11}+\pi_{13}+\pi_{31}+\pi_{33}+2 \pi_{44}\right) \sigma d_{\overline{4}} n_{4}^{3}+\frac{1}{4}\left(S_{11}+2 S_{13}+S_{33}-S_{44}\right) \sigma d_{\overline{4}}\left(n_{4}-1\right) .
$$

In the frame of half-wave stress technique we have $\delta \Delta_{\overline{4}}=\lambda / 2$, with $\sigma=\sigma_{i m}=\sigma_{44}$ being the half-wave stress and $\sigma_{44}^{o}=\sigma_{44} d_{\overline{4}}$ the operating stress. Let us insert these relations into Eq. (2) and take into account the conditions needed for compensation of the error caused by the wedge-like shape of sample (in a manner analogical to Eq. (1), one should replace $1 / \sigma_{i m}^{o}$ with $\left(1 / \sigma_{i m}^{o}+1 / \sigma_{i m}^{\prime o}\right) / 2$. Then one gets the relation

$$
\pi_{11}+\pi_{13}+\pi_{31}+\pi_{33}+2 \pi_{44}=-\frac{2 \lambda}{n_{4}^{3}}\left(\frac{1}{\sigma_{44}^{o}}+\frac{1}{\sigma_{44}^{\prime O}}\right)+2\left(S_{11}+2 S_{13}+S_{33}-S_{44}\right) \frac{n_{4}-1}{n_{4}^{3}},
$$

which can be used for evaluating the coefficient $\pi_{44}$ on the basis of experimentally determined operating stresses $\sigma_{44}^{o}$ and $\sigma_{44}^{\prime o}$. In case when so-called 'symmetric' experimental conditions are dealt with $(i=\overline{4}, k=4$ and $m=\overline{4})$, one obtains the relation analogical to Eq. (3), where the parameters $\sigma_{44}^{o}$ and $\sigma_{44}^{\prime o}$ are replaced by $\sigma_{\overline{4}}^{o}$ and $\sigma_{\frac{\prime}{4}}^{\prime o}$ (see Eq. (T.1) in Table 1).

Below we will consider a number of experimental geometries relying on a $X / 45^{\circ}$-cut sample (see Fig. 1b), which are used for checking reliability of the POCs values and signs. Basing upon the example of crystals belonging to the point symmetry classes 32 and $3 \mathrm{~m}$, the authors of the works $[9,10]$ have demonstrated for the first time that the $X / 45^{\circ}$-cut sample allows for determining the principal coefficient $\pi_{11}$ in two different experimental geometries. The coefficient $\pi_{11}$ for the classes 
$4, \overline{4}$ and $4 / \mathrm{m}$ can also be determined using the $X / 45^{\circ}$-cut sample (notice that derivation of the corresponding theoretical relations is the same as in Refs. $[9,10])$. The relevant relations valid for the direct and symmetric experiment geometries are gathered in Table 1 (see Eq. (T.2)). Notice also that the $X / 45^{\circ}$-cut sample admits experimental geometries, for which the sums of principal POCs can be found. Then a comparison of these sums with the corresponding sums arising from the coefficient values determined independently on the direct-cut samples (see Fig. 1a) would serve as a reliability criterion for the POCs.

Let us consider the experimental geometry given by $m=4, k=\overline{4}$ and $i=1$. The relation $\delta n_{1}=-\delta B_{1} n_{1}^{3} / 2$, which can easily be obtained after differentiating the optical impermeability coefficients $B_{i}=1 / n_{\mathrm{i}}^{2}$, is valid for the light polarization direction $i=1$. Let us evaluate the change $\delta B_{1}$ in the optical impermeability coefficient, using the equation of the POE

$$
\delta B_{i}=\pi_{i m} \sigma_{m} .
$$

The summation in Eq. (4) is performed over the index $m=1,2, \ldots, 6$, and $\sigma_{m}$ denote the mechanical stress tensor components.

Now it is necessary to find the form of the stress tensor $\left[\sigma_{m}\right]$ on the basis of the relation [14]

$$
\left[\sigma_{m}\right]=\left[\sigma_{1}, \sigma_{2}, \sigma_{3}, \sigma_{4}, \sigma_{5}, \sigma_{6}\right]=\left[a^{2}, b^{2}, c^{2}, b c, a c, a b\right] \sigma,
$$

where $a, b, c$ are the directional cosines of the uniaxial loading force vector $\boldsymbol{P}, \sigma$ is the value uniaxial pressure. Obviously, if the force acts along the direction $m=4$ (see Fig. 1b), then we have $a=b=\cos 45^{\circ}=\sqrt{2} / 2$ and $c=\cos 90^{\circ}=0$. In terms of Eq. (5), the $\left[\sigma_{4}\right]$ tensor (for $m=4$ ) may be given as

$$
\left[\sigma_{4}\right]=\sigma\left[0, \frac{1}{2}, \frac{1}{2}, \frac{1}{2}, 0,0\right] .
$$

Hence, the non-zero components of the tensor $\left[\sigma_{4}\right]$ are as follows: $\sigma_{2}=\sigma_{3}=\sigma_{4}=\sigma / 2$. Using

Eq. (4) and the matrix of POCs for the symmetry classes $4, \overline{4}$ and $4 / \mathrm{m}[8,13]$, one gets

$$
\delta B_{1}=\frac{1}{2}\left(\pi_{12}+\pi_{13}\right) \sigma .
$$

Thence the relation for $\delta n_{1}$ follows:

$$
\delta n_{1}=-\frac{1}{4}\left(\pi_{12}+\pi_{13}\right) \sigma n_{1}^{3} .
$$

One has to insert this relation into the formulae describing the change in the optical path for the light propagating though sample [14]:

$$
\delta \Delta_{k}=\delta n_{i} d_{k}+\varepsilon_{k}^{*} d_{k}\left(n_{i}-1\right),
$$

where $\varepsilon_{k}^{*}$ means the strain occurring along the direction $k$ of light propagation. Here the star indicates that the strain $\varepsilon_{k}^{*}$ in the $k$-direction differs from the components of the strain tensor $\varepsilon_{k}$.

To derive the sample strain $\varepsilon_{\overline{4}}^{*}$ along the direction $k=\overline{4}$ (see [14]), we are to consider a cross section of a surface of strain tensor,

$$
\varepsilon_{1} x_{1}^{2}+\varepsilon_{2} x_{2}^{2}+\varepsilon_{3} x_{3}^{2}+\varepsilon_{4} x_{2} x_{3}+\varepsilon_{5} x_{1} x_{3}+\varepsilon_{6} x_{1} x_{2}=1
$$

by a line given by $x_{1}=0$ and $x_{3}=-x_{2}$, which corresponds to the optical beam direction $k=\overline{4}$ (see Fig. 2): 


$$
\varepsilon_{2} x_{2}^{2}+\varepsilon_{3} x_{2}^{2}-\varepsilon_{4} x_{2}^{2}=1
$$

with $\varepsilon_{2}, \varepsilon_{3}, \varepsilon_{4}$ being the components of the strain tensor. Using Eq. (11), one can find the coordinate $x_{2}$ corresponding to the cross section point of the line $k=\overline{4}$ and the surface determined by Eq. (10):

$$
x_{2}=\frac{1}{\sqrt{\varepsilon_{2}+\varepsilon_{3}-\varepsilon_{4}}} .
$$

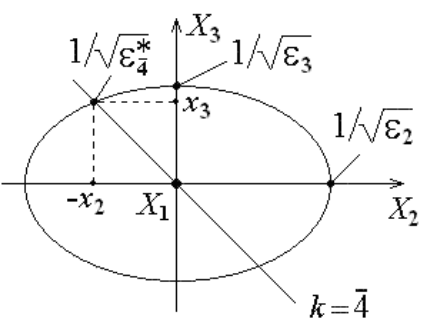

Fig. 2. Cross section of characteristic surface of the strain tensor by a line $k=\overline{4}$ (see the text): $x_{1}=0, x_{3}=-x_{2}$.

The semi-axis of the surface given by Eq. (10) is determined as $e_{\frac{*}{4}}^{*}=1 / \sqrt{\varepsilon_{\frac{*}{4}}^{*}}$ (see Fig. 2 and [13, 14]). From the other side, one can find the semi-axis value as $e_{4}^{*}=\sqrt{x_{2}^{2}+x_{3}^{2}}=x_{2} \sqrt{2}$. After accounting for Eq. (12), one gets

$$
e_{\overline{4}} *=\frac{1}{\sqrt{\varepsilon_{\overline{4}} *}}=x_{2} \sqrt{2}=\frac{\sqrt{2}}{\sqrt{\varepsilon_{2}+\varepsilon_{3}-\varepsilon_{4}}},
$$

The latter yields in the following sample strain occurring along the direction $k=\overline{4}$ :

$$
\varepsilon_{\frac{1}{4}}^{*}=\frac{1}{2}\left(\varepsilon_{2}+\varepsilon_{3}-\varepsilon_{4}\right) \text {. }
$$

The components $\varepsilon_{2}, \varepsilon_{3}$ and $\varepsilon_{4}$ of the strain tensor are to be determined issuing from the Hook's law ( $\varepsilon_{k}=S_{k m} \sigma_{m}$ ) and using the elastic compliance matrix $S_{k m}[8,13]$ for the classes $4, \overline{4}$ and $4 / \mathrm{m}$, and the stress tensor components $\left[\sigma_{4}\right]$ from Eq. (6) $\left(\varepsilon_{2}=\left(S_{11}-S_{13}\right) \sigma / 2, \varepsilon_{3}=\left(S_{13}-S_{33}\right) \sigma / 2\right.$, and $\left.\varepsilon_{2}=S_{44} \sigma / 2\right)$. Inserting these relations into Eq. (13), one arrives at the strain occurring along the direction $k=\overline{4}$ under the action of uniaxial stress along the direction $m=4$ :

$$
\varepsilon_{4}^{*}=\frac{\sigma}{4}\left(S_{11}+2 S_{13}+S_{33}-S_{44}\right) .
$$

Basing on Eqs. (14), (8) and (9), one can derive the sum of the principal POCs $\pi_{12}+\pi_{13}$ for the experimental geometry of $X / 45^{\circ}$-cut sample (the optical path change $\delta \Delta_{k}$ ) with accounting for the Poisson strain:

$$
\delta \Delta_{\overline{4}}=-\frac{1}{4}\left(\pi_{12}+\pi_{13}\right) \sigma d_{\overline{4}} n_{1}^{3}+\frac{1}{4}\left(S_{11}+2 S_{13}+S_{33}-S_{44}\right) \sigma d_{\overline{4}}\left(n_{1}-1\right) .
$$

This may be specified for the cases of half-wave stress technique and wedge-like sample shape as

$$
\pi_{12}+\pi_{13}=-\frac{\lambda}{n_{1}^{3}}\left(\frac{1}{\sigma_{14}^{o}}+\frac{1}{\sigma_{14}^{\prime o}}\right)+\left(S_{11}+2 S_{13}+S_{33}-S_{44}\right) \frac{n_{1}-1}{n_{1}^{3}} .
$$


The relation for the symmetric experimental conditions of determining the sum $\pi_{12}+\pi_{13}(m=\overline{4}$, $k=4$, and $i=1$ ) are analogical and can be obtained after substituting the operating stresses $\sigma_{14}^{o}, \sigma_{14}^{\prime o}$ by $\sigma_{1 \overline{4}}^{o}, \sigma_{1 \frac{0}{4}}^{\prime}$. These two relations are presented in Table 1 (see Eq. (T.3)).

Notice that the $X / 45^{\circ}$-cut sample also allows accomplishing the additional experimental geometries. In particular, the direct conditions given by $m=1, k=\overline{4}$ and $i=4$, and the symmetrical ones ( $m=1, k=4$ and $i=\overline{4}$ ) enable finding the sum $\pi_{12}+\pi_{31}$; the pair of direct and symmetrical (in brackets) conditions specified by $m=4(\overline{4}), k=1$ and $i=2$ can result in the sum $\pi_{11}+\pi_{13}$; and the conditions $m=4(\overline{4}), k=1$ and $i=3$ yield in the sum $\pi_{31}+\pi_{33}$. All the analytical relations for the $X / 45^{\circ}$-cut samples are given in Table 1 by Eqs. (T.1)-(T.6), two of which are symmetrically identical to Eq. (T.1). These relations facilitate determining the coefficient $\pi_{44}$ for the both direct and symmetrical experimental conditions. Basing on the other ten relations, one can find the coefficient $\pi_{11}$ and the other sums of the principal POCs.

The direct and symmetric experimental conditions differ by the directions of light propagation and polarization ( 4 or $\overline{4})$, as well as by the directions of mechanical loading. Table 1 shows that all of the relations for the direct and symmetric conditions are in fact the same, i.e. the results for the POCs or their sums are independent of choice of the directions 4 or $\overline{4}$. As a consequence, one can define these directions, along with the corresponding experimental conditions, as 'symmetrically identical'. Below we will show that, for the groups of symmetry $4, \overline{4}$ and $4 / \mathrm{m}$, the directions 6 and $\overline{6}$ (for a $Z / 45^{\circ}$-cut sample) and the directions $6^{*}$ and $\overline{6}^{*}$ (for a $Z / 22.5^{\circ}$-cut sample - see Fig. 1c, d) are also symmetrically identical. Thus, one can correctly and unambiguously determine the non-principal POCs irrespective of solving the problem of definition of these directions. One should emphasise in this respect that the analysis [14] has proved the directions 4 and $\overline{4}$ to be not symmetrically identical for the trigonal symmetry groups $3, \overline{3}, 3 \mathrm{~m}$ and 32 . Relevant recommendations for unambiguous choice of these directions have also been formulated in this work.

\section{Experimental manifestations of POE for $Z / 45^{\circ}$-, $X / 22.5^{\circ}$ - and $B$-cut samples}

Above we have considered in detail how to derive the theoretical relations for the POE associated with the $X / 45^{\circ}$-cut sample. The final relations used for determining the non-principal POCs for the samples of other orientations are presented in Table 1. Each line of Table 1 combines the two relations referred to the direct and symmetric experimental conditions, the latter being put in brackets. The main peculiarities of these relations will be discussed below and the appropriate practical conclusions will be drawn.

\subsection{Z/45 ${ }^{\circ}$-cut sample}

Taking the conditions $i=3, m=3$ and $k=1$ (or $k=2$ - see Fig. 1) in Eq. (1), one can obtain the following relation for determination of the coefficient $\pi_{33}$ :

$$
\pi_{33}=-\frac{\lambda}{2 n_{3}^{3}}\left(\frac{1}{\sigma_{33}^{o}}+\frac{1}{\sigma_{33}^{o o}}\right)+\frac{2 S_{13}}{n_{3}^{3}}\left(n_{3}-1\right)
$$

where the condition $S_{13}=S_{23}$ holds true. A comparison of Eq. (T.7) for the $Z / 45^{\circ}$-cut sample (see Table 1) with Eq. (17) for the direct-cut sample testifies that these relations are the same.

Now let us write out the relations for the coefficient $\pi_{13}$ under the conditions $i=1, m=3, k=2$ and $i=2, m=3, k=1$, following from Eq. (1): 


$$
\begin{aligned}
& \pi_{13}=-\frac{\lambda}{2 n_{1}^{3}}\left(\frac{1}{\sigma_{13}^{o}}+\frac{1}{\sigma_{13}^{\prime o}}\right)+\frac{2 S_{13}}{n_{1}^{3}}\left(n_{1}-1\right), \\
& \pi_{23}=-\frac{\lambda}{2 n_{1}^{3}}\left(\frac{1}{\sigma_{23}^{o}}+\frac{1}{\sigma_{23}^{\prime o}}\right)+\frac{2 S_{13}}{n_{1}^{3}}\left(n_{1}-1\right) .
\end{aligned}
$$

After comparing these equations with each other and with Eqs. (T.8), and taking into account that the equality $\pi_{13}=\pi_{23}$ is satisfied for the symmetry classes under test, one arrive at the conclusion that all of these relations are identical. The operating stresses are also the same $\left(\sigma_{13}^{o}=\sigma_{23}^{o}=\sigma_{63}^{o}=\sigma_{63}^{o}\right)$. However, the latter equalities cannot be true in case when the samples have a micro-wedge shape. Nonetheless, the following equalities remain to be valid for the sums of the $\sigma_{i m}^{o}$ reciprocals in the latter case:

$$
\frac{1}{\sigma_{13}^{o}}+\frac{1}{\sigma_{13}^{\prime o}}=\frac{1}{\sigma_{23}^{o}}+\frac{1}{\sigma_{23}^{\prime o}}=\frac{1}{\sigma_{63}^{o}}+\frac{1}{\sigma_{63}^{\prime o}}=\frac{1}{\sigma_{63}^{o}}+\frac{1}{\sigma_{63}^{\prime o}} .
$$

We should also stress that the $Z / 45^{\circ}$-cut sample allows for four different experimental geometries for measuring the rotational-shifting coefficient $\pi_{66}$. These geometries are described by Eqs. (T.9) and (T.11). Nonetheless, Eqs. (T.11) remain to be preferable. Indeed, the corresponding experimental error for the POC $\pi_{66}$ is then associated only with the error of determining the operating stresses, while utilisation of Eqs. (T.9) will give rise to additional errors referred to determination of the Poisson-contribution term and to the principal POCs $\pi_{11}$ and $\pi_{12}$.

\section{2. $Z / 22.5^{0}$-cut sample}

Let us write out the analytical relation for determination of the sum of POCs $\pi_{16}+\pi_{61}$ [14]:

$$
\begin{aligned}
\delta \Delta_{\overline{6}^{*}} & =-\frac{1}{2} \sigma d_{\overline{6}^{*}} n_{1}^{3} \cos ^{4} \alpha \\
& \times\left[\pi_{11}\left(1+\tan ^{4} \alpha\right)+2\left(\pi_{12}+\pi_{66}\right) \tan ^{2} \alpha+\left(\pi_{16}+2 \pi_{61}\right)\left(\tan ^{3} \alpha-\tan \alpha\right)\right] \\
& +\sigma d_{\overline{6}^{*}} \cos ^{4} \alpha\left[\left(2 S_{11}-S_{66}\right) \tan ^{2} \alpha+S_{12}\left(1+\tan ^{4} \alpha\right)-2 S_{16}\left(\tan ^{3} \alpha-\tan \alpha\right)\right]\left(n_{1}-1\right),
\end{aligned}
$$

where the half-wave stress technique and the consideration of a wedge-like sample shape are intended, as always. Let us substitute $\delta \Delta_{\overline{6}^{*}}$ in Eq. (21) with $\lambda / 2$ and $\sigma$ with $\sigma_{6^{*} 6^{*}}^{o}$ (see Eqs. (2) and (3) and the relevant comments). Then the following relations for the sums $\pi_{16}+2 \pi_{61}$ expressed in terms of the operating stresses $\sigma_{6^{*} 6^{*}}^{o}$ and $\sigma_{66^{*}}^{\prime O}$ will take place:

$$
\begin{aligned}
& \pi_{11}\left(1+\tan ^{4} \alpha\right)+2\left(\pi_{12}+\pi_{66}\right) \tan ^{2} \alpha+\left(\pi_{16}+2 \pi_{61}\right)\left(\tan ^{3} \alpha-\tan \alpha\right) \\
& =-\frac{\lambda}{2 n_{1}^{3} \cos ^{4} \alpha}\left(\frac{1}{\sigma_{6^{*} 6^{*}}^{o}}+\frac{1}{\sigma_{6^{*} 6^{*}}^{o}}\right)+2\left[\left(2 S_{11}-S_{66}\right) \tan ^{2} \alpha\right. \\
& \left.+S_{12}\left(1+\tan ^{4} \alpha\right)-2 S_{16}\left(\tan ^{3} \alpha-\tan \alpha\right)\right]\left(n_{1}-1\right) / n_{1}^{3} .
\end{aligned}
$$

This formula is valid for the experimental conditions $i=m=6^{*}$ and $k=\overline{6}^{*}$.

The authors of the work [14], from which Eq. (21) is taken, have not analysed the symmetric experimental conditions $i=m=\overline{6}^{*}$ and $k=6^{*}$. Therefore it is not clear whether the ambiguity in determination of the sum $\pi_{16}+2 \pi_{61}$ exists for the direct and symmetric conditions. Let us consider the relations for the symmetric conditions analogical to Eqs. (21) and (22). For this aim we should 
establish the form of the stress tensor for the case of $m=\overline{6}^{*}$. According to Fig. 3, the directional cosines $a, b, c$ of the vector $\boldsymbol{P}\left(m=\overline{6}^{*}\right)$ may be found as

$$
\boldsymbol{P}=\boldsymbol{P}(a, b, c)=\boldsymbol{P}(\cos \alpha,-\sin \alpha, 0) .
$$

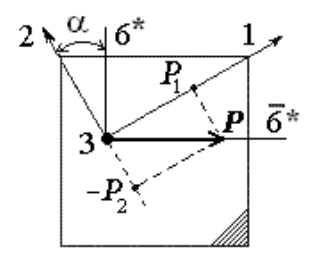

Fig. 3. A scheme explaining determination of directional cosines for the vector $\boldsymbol{P}$ (see the text).

Combining Eq. (23) and Eq. (5), one can derive the components of $\left[\sigma_{\overline{6}^{*}}\right]$ tensor:

$$
\begin{aligned}
& {\left[\sigma_{\bar{\sigma}^{*}}\right]=\sigma\left[\cos ^{2} \alpha, \sin ^{2} \alpha, 0,0,0,-\sin \alpha \cos \alpha\right]} \\
& =\sigma \cos ^{2} \alpha\left[1, \tan ^{2} \alpha, 0,0,0,-\tan \alpha\right] .
\end{aligned}
$$

To determine the refractive index change $\delta n_{i}=\delta n_{\overline{6}^{*}}$, one should intersect the optical indicatrix by the line $i=\overline{6}^{*}$ (the equation of line $x_{2}=-x_{1} \tan \alpha$ and $x_{3}=0$ - see Fig. 4) and use the equation of the perturbed indicatrix [14]):

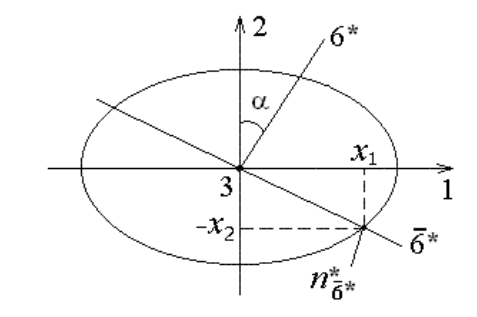

Fig. 4. Cross section of perturbed optical indicatrix by a line $i=\overline{6}^{*}$ (see the text).

$$
\left(B_{1}+\delta B_{1}\right) x_{1}^{2}+\left(B_{2}+\delta B_{2}\right) x_{2}^{2}+\left(B_{3}+\delta B_{3}\right) x_{3}^{2}+2 \delta B_{4} x_{2} x_{3}+2 \delta B_{5} x_{1} x_{3}+2 \delta B_{6} x_{1} x_{2}=1 .
$$

The result is

$$
\left(B_{1}+\delta B_{1}\right) x_{1}^{2}+\left(B_{2}+\delta B_{2}\right) x_{1}^{2} \tan ^{2} \alpha-2 \delta B_{6} x_{1}^{2} \tan \alpha=1,
$$

or

$$
x_{1}=\frac{1}{\sqrt{B_{1}+B_{2} \tan ^{2} \alpha+\delta B}}, \quad \delta B=\delta B_{1}+\delta B_{2} \tan ^{2} \alpha-2 \delta B_{6} \tan \alpha .
$$

The refractive index $n_{\overline{6}^{*}} *$ is equal to (see Fig. 4)

$$
n_{\overline{6}^{*}} *=\sqrt{x_{1}^{2}+x_{2}^{2}}=x_{1} \sqrt{1+\tan ^{2} \alpha}=x_{1} / \cos \alpha
$$

or, while considering Eq. (26) and the equality $B_{1}=B_{2}=1 / n_{1}^{2}$, we have

$$
\begin{aligned}
& n_{\bar{\sigma}^{*}} *=\frac{1}{\cos \alpha} \frac{1}{\sqrt{B_{1}+B_{2} \tan ^{2} \alpha+\delta B}}=\frac{1}{\cos \alpha} \frac{1}{\sqrt{B_{1}\left(1+\tan ^{2} \alpha\right)+\delta B}} \\
& =\frac{n_{1}}{\cos \alpha} \frac{1}{\sqrt{1+\tan ^{2} \alpha+n_{1}^{2} \delta B}}=\frac{n_{1}}{\sqrt{1+n_{1}^{2} \delta B \cos ^{2} \alpha}} .
\end{aligned}
$$

The same refractive index under the condition of non-perturbed indicatrix (see Eq. (27) and the conditions $\delta B=0$ ) is equal to $n_{\overline{6}^{*}}=n_{1}$. Then the change in the refractive index equals to $\delta n_{\overline{6}^{*}} *=n_{\overline{6}^{*}} *-n_{1}$, i.e. we have 


$$
\delta n_{\overline{6}^{*}} *=\frac{n_{1}}{\sqrt{1+n_{1}^{2} \delta B \cos ^{2} \alpha}}-n_{1}=-\frac{1}{2} n_{1}^{2} \delta B \cos ^{2} \alpha .
$$

The relations for the $\delta B_{i}$ parameters $(i=1,2,6)$ follow from the form of the POC matrix and the tensor $\left[\sigma_{\overline{6}^{*}}\right]$, as well as Eqs. $(24),(26)$ and (4):

$$
\begin{aligned}
& \delta B_{1}=\sigma \cos ^{2} \alpha\left(\pi_{11}+\pi_{12} \tan ^{2} \alpha-\pi_{16} \tan \alpha\right), \\
& \delta B_{2}=\sigma \cos ^{2} \alpha\left(\pi_{12}+\pi_{11} \tan ^{2} \alpha+\pi_{16} \tan \alpha\right), \\
& \delta B_{6}=\sigma \cos ^{2} \alpha\left(\pi_{61}-\pi_{61} \tan ^{2} \alpha-\pi_{66} \tan \alpha\right) .
\end{aligned}
$$

With Eqs. (26) and (28) this leads to

$$
\delta n_{\overline{6}^{*}} *=-\frac{1}{2} n_{1}^{3} \sigma \cos ^{4} \alpha\left[\pi_{11}\left(1+\tan ^{4} \alpha\right)+2\left(\pi_{12}+\pi_{66}\right) \tan ^{2} \alpha+\left(\pi_{16}+2 \pi_{61}\right)\left(\tan ^{3} \alpha-\tan \alpha\right)\right] .
$$

The sample strain $\varepsilon_{6^{*}} *\left(k=6^{*}\right)$ under the action of the stress tensor $\left[\sigma_{\overline{6}^{*}}\right]$ (see Eq. $(24)$ and Fig. 5) can be found after intersecting the characteristic surface by the line given by the formulae $x_{1}=x_{2} \tan \alpha$ and $x_{3}=0$ (see Eq. (10)):

$$
\varepsilon_{1} x_{2}^{2} \tan ^{2} \alpha+\varepsilon_{2} x_{2}^{2}+\varepsilon_{6} x_{2}^{2} \tan \alpha=1, x_{2}=\frac{1}{\sqrt{\varepsilon_{1} \tan ^{2} \alpha+\varepsilon_{2}+\varepsilon_{6} \tan \alpha}} .
$$

It is seen from Fig. 5 that the semi-axis $1 / \sqrt{\varepsilon_{6^{*}} *}$ reads as

$$
\frac{1}{\sqrt{\varepsilon_{6^{*}}^{*}}}=\sqrt{x_{1}^{2}+x_{2}^{2}}=x_{2} \sqrt{1+\tan ^{2} \alpha}=\frac{x_{2}}{\cos \alpha}=\frac{1}{\cos \alpha} \cdot \frac{1}{\sqrt{\varepsilon_{1} \tan ^{2} \alpha+\varepsilon_{2}+\varepsilon_{6} \tan \alpha}},
$$

or

$$
\varepsilon_{6^{*}} *=\cos ^{2} \alpha\left(\varepsilon_{1} \tan ^{2} \alpha+\varepsilon_{2}+\varepsilon_{6} \tan \alpha\right)
$$

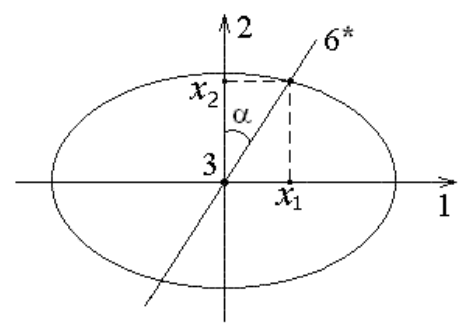

Fig. 5. Cross section of characteristic surface of the strain tensor by a line $k=6^{*}$ (see the text).

Finally, the strain tensor components $\varepsilon_{1}, \varepsilon_{2}$ and $\varepsilon_{6}$ may be obtained from the Hook's law, the form of the $\left[\sigma_{\overline{6}^{*}}\right]$ tensor (see Eq. (24)), and the elastic compliance matrix $S_{k m}$ :

$$
\begin{aligned}
& \varepsilon_{1} \tan ^{2} \alpha=\sigma \cos ^{2} \alpha\left(S_{11} \tan ^{2} \alpha+S_{12} \tan ^{4} \alpha-S_{16} \tan ^{3} \alpha\right), \\
& \varepsilon_{2}=\sigma \cos ^{2} \alpha\left(S_{12}+S_{11} \tan ^{2} \alpha+S_{16} \tan \alpha\right), \\
& \varepsilon_{6} \tan \alpha=\sigma \cos ^{2} \alpha\left(S_{16} \tan \alpha-S_{16} \tan ^{3} \alpha-S_{66} \tan ^{2} \alpha\right) .
\end{aligned}
$$

Basing on Eqs. (29), (31), (32) and (9), one gets

$$
\begin{aligned}
& \delta \Delta_{6^{*}}=-\frac{1}{2} \cos ^{4} \alpha\left[\pi_{11}\left(1+\tan ^{4} \alpha\right)+2\left(\pi_{12}+\pi_{66}\right) \tan ^{2} \alpha+\left(\pi_{16}+2 \pi_{61}\right) \times\left(\tan ^{3} \alpha-\tan \alpha\right)\right] d_{6^{*}} \sigma n_{1}^{3} \\
& +\cos ^{4} \alpha\left[\left(2 S_{11}-S_{66}\right) \tan ^{2} \alpha+S_{12}\left(1+\tan ^{4} \alpha\right)-2 S_{16}\left(\tan ^{3} \alpha-\tan \alpha\right)\right] d_{6^{*}} \sigma\left(n_{1}-1\right) .
\end{aligned}
$$

Notice that Eq. (33) is identical to Eq. (21). Hence, we have the two independent relations for de- 
termination of the POC sum $\pi_{16}+2 \pi_{61}$ in the two symmetrically identical experimental conditions:

$$
i=m=6^{*}, k=\overline{6}^{*}, \quad i=m=\overline{6}^{*}, k=6^{*} .
$$

Another principled question is as follows: do the relations for $\delta \Delta_{6^{*}}$ linked to the $Z / 22.5^{\circ}$-cut sample (see Eqs. (21) and (33) and Fig. 3) depend on the choice of right-handed coordinate system and, respectively, on the choice of directions $6^{*}$ and $\overline{6}^{*}$ ? For example, let us rotate the coordinate system around the axis 1 by $180^{\circ}$ (see Fig. 3). Under such conditions the directions $6^{*}$ and $\overline{6}^{*}$ are interchanged. However, the sums of the POCs $\pi_{16}+2 \pi_{61}$ are given by the identical relations in the both cases, as shown for the particular conditions (34) differing just by the directions $6^{*}$ and $\overline{6}^{*}$. One can also demonstrate a possibility for arbitrary choice of the directions $6^{*}$ and $\overline{6}^{*}$ for the most general case. For this aim let us rotate a sample shown in Fig. 3 by $90^{\circ}$ around the axis 3 (see Fig. 6). This will result in replacing the axis 1 by the axis 2, and vice versa. Indeed, in tetragonal crystals these axes are identical with respect to both the refractive indices and the POCs. As a result, we introduce the angle $\alpha$ and the directions $6^{*}$ and $\overline{6}^{*}$ the same as in Fig. 3.

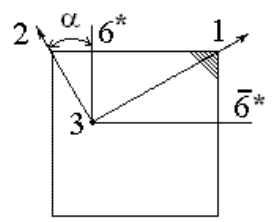

Fig. 6. A sample rotated around the axis 3 by $90^{\circ}$ with respect to that shown in Fig. 3 (see the text).

Thus we have proved that the sum $\pi_{16}+2 \pi_{61}$ is unambiguously determined for the case of $Z / 22.5^{\circ}$-cut samples. The corresponding Eqs. (21) and (33) are now combined into Eq. (T.15) in Table 1 , which can be used in the frame of half-wave stress technique with taking into account microwedge sample shapes.

Notice that the relations (33) and (T.15) at $\alpha=45^{\circ}$ are transformed into Eqs. (T.9) for the $Z / 45^{\circ}$ cut sample, which do not contain the sum $\pi_{16}+2 \pi_{61}$. Using the samples of $Z / 22.5^{\circ}$-cut, it is possible to determine the coefficients $\pi_{33}, \pi_{13}$ and $\pi_{31}$, as well as the sum $\pi_{11}+\pi_{12}$ (see Table 1 and Eqs. (T.13), (T.14), (T.16), (T.21) and (T.22)). Eqs. (T.13) and (T.14) for determination of the coefficients $\pi_{33}$ and $\pi_{13}$ do not depend on the angle $\alpha$. They are identical both to Eqs. (T.7) and (T.8) for the $\mathrm{Z} / 45^{\circ}$-cut samples and to the corresponding relations obtained for the direct-cut samples (see Eqs. (17)-(19)).

Eq. (T.16) includes a complicated sum of the elastic compliance coefficients $S_{k m}\left(\Sigma S_{k m}\right)$. The latter can be derived while inserting the expression for the coefficient $\pi_{31}$ into this relation (notice that the $\pi_{31}$ coefficient is determined using the direct-cut sample). Inserting the sum $\Sigma S_{k m}$ into Eq. (T.15) enables finding the sum $\pi_{16}+2 \pi_{61}$ with essentially reduced error. Eqs. (T.19) and (T.20) are also important because they do not contain the Poisson strain contribution and thus the experimental errors for the sum $\pi_{16}+2 \pi_{61}$ can be small enough.

\subsection{B-cut sample}

\subsubsection{General considerations}

In order to describe completely the POE for the classes $4, \overline{4}$ and $4 / \mathrm{m}$, we are to derive the relations used when determining the shifting coefficient $\pi_{16}$ and the rotational-shifting coefficient $\pi_{45}$. To determine the POC $\pi_{16}$, it is necessary to prepare a sample, which ensures that the light 
is polarised along the direction $i=1$ and the component $\sigma_{6}$ of the mechanical stress tensor $\sigma_{m}$ remains nonzero. Determination of $\pi_{45}$ means utilisation of a sample allowing to provide the light polarization $i=4$ and a nonzero component $\sigma_{5}$.

We have found out that these conditions can be provided with a sample of so-called $B$-cut. As seen from the scheme of such a sample given by Fig. 7, one has to make cuts at the angles of $45^{\circ}$ with respect to the directions 1 and 4 , using the initial $X / 45^{\circ}$-cut sample (the planes of the cuts are shown by dotted lines in Fig. 7; see also Fig. 1e for more details).

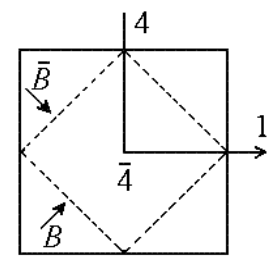

Fig. 7. A scheme of $B$-cut sample for determining $\pi_{16}$ and $\pi_{45}$ coefficients; $B$ and $\bar{B}$ faces are indicated by arrows (see the text).

Fig. 7 testifies that, with the light propagating along the direction $k=\overline{4}$, it is possible to ensure the polarization parallel to the $i=1$ and $i=4$ directions. For finding the changes in the refractive indices $\delta n_{1}$ and $\delta n_{4}$, as well as the strain $\varepsilon_{\overline{4}} *$ occurring along the direction $k=\overline{4}$, one should obtain the stress tensor for the case when the uniaxial pressure force $\boldsymbol{P}$ is applied along the directions perpendicular to the faces $B$ and $\bar{B}$ (i.e., $m \perp B$ and $m \perp \bar{B}$ ).

It is easy to show that the directional cosines $a, b, c$ of the vector $\boldsymbol{P}$ under the condition of $m \perp B$ are as follows:

$$
P_{B}=P_{B}(a, b, c)=P_{B}\left(\cos 45^{\circ}, \cos 60^{\circ}, \cos 60^{\circ}\right)=P_{B}(\sqrt{2} / 2,1 / 2,1 / 2),
$$

while under the condition of $m \perp \bar{B}$ one has

$$
P_{\bar{B}}=P_{\bar{B}}\left(\cos 45^{\circ},-\cos 60^{\circ},-\cos 60^{\circ}\right)=P_{\bar{B}}(\sqrt{2} / 2,-1 / 2,-1 / 2) .
$$

According to Eq. (5), the stress tensors for the cases of $m \perp B$ and $m \perp \bar{B}$ are given by

$$
\begin{aligned}
& {\left[\sigma_{B}\right]=\sigma\left[\frac{1}{2}, \frac{1}{4}, \frac{1}{4}, \frac{1}{4}, \frac{\sqrt{2}}{4}, \frac{\sqrt{2}}{4}\right],} \\
& {\left[\sigma_{\bar{B}}\right]=\sigma\left[\frac{1}{2}, \frac{1}{4}, \frac{1}{4}, \frac{1}{4},-\frac{\sqrt{2}}{4},-\frac{\sqrt{2}}{4}\right] .}
\end{aligned}
$$

\subsubsection{Independent determination of the rotational coefficient $\pi_{16}$}

The conditions needed for determining the $\pi_{16}$ coefficient are as follows: $i=1, k=\overline{4}$, and $m \perp B$. After calculating the refractive index change $\delta n_{1}$ and the sample strain $\varepsilon_{\overline{4}} *$ and considering Eq. (35), one can find the optical path change $\delta \Delta_{\overline{4}}$ on the basis of Eq. (9). When $m \perp B$, we obtain

$$
\begin{aligned}
\delta \Delta_{\overline{4}}= & -\frac{1}{8}\left(2 \pi_{11}+\pi_{12}+\pi_{13}+\sqrt{2} \pi_{16}\right) \sigma d_{\overline{4}} n_{1}^{3} \\
& +\frac{1}{8}\left(S_{11}+S_{33}+2 S_{12}+4 S_{13}-S_{44}-\sqrt{2} S_{16}\right) \sigma d_{\overline{4}}\left(n_{1}-1\right) .
\end{aligned}
$$

The uniaxial pressure direction parallel to $m \perp \bar{B}$ would lead to the sign reversal for the components $\sigma_{5}$ and $\sigma_{6}$ (see Eq. (36)). Then the relation allowing determination of the POC $\pi_{16}$ will differ by the signs of the terms $\sqrt{2} \pi_{16}$ and $\sqrt{2} S_{16}$ : 


$$
\begin{aligned}
\delta \Delta_{\overline{4}}= & -\frac{1}{8}\left(2 \pi_{11}+\pi_{12}+\pi_{13}-\sqrt{2} \pi_{16}\right) d_{\overline{4}} \sigma n_{1}^{3} \\
& +\frac{1}{8}\left(S_{11}+S_{33}+2 S_{12}+4 S_{13}-S_{44}+\sqrt{2} S_{16}\right) d_{\overline{4}} \sigma\left(n_{1}-1\right) .
\end{aligned}
$$

Eqs. (37) and (38) that take the micro-wedge shape of samples into consideration and can be used with the half-wave technique are included in Table 1 (see Eq. (T.23)). Notice that, apart of $\pi_{16}$, Eq. (T.23) contains the four principal POCs and a complicated sum of the $S_{k m}$ coefficients. Potentially this can bring about increasing errors and, as a result, low experimental accuracy for the $\pi_{16}$ coefficient. On the other hand, the signs of the $\pi_{16}$ coefficient in the two versions of Eq. (T.23) written for the direct and symmetric experimental conditions are opposite. Then one can exclude all the principal POCs and all the elastic compliances, except for $S_{16}$, when using the difference between these versions of Eqs. (T.23) written under the conditions $m \perp \bar{B}$ and $m \perp B$. As a consequence, one derives a simple relation given by Eq. (T.24), which is preferable while calculating the $\operatorname{POC} \pi_{16}$.

\subsubsection{Determination of the POC difference $2 \pi_{45}-\pi_{16}$}

Under the conditions considered above $(i=1, k=\overline{4}$ and $m \perp B)$ it is necessary to change the direction of light polarization by $90^{\circ}$, resulting in $i=4$. Calculating $\delta n_{4}$ and $\varepsilon_{\overline{4}}^{*}$ with the procedures described above and inserting these parameters into Eq. (9), one can obtain the relation that includes the POC $\pi_{45}$ :

$$
\begin{aligned}
& \delta \Delta_{\overline{4}}=-\frac{1}{16}\left[\pi_{11}+\pi_{13}+\pi_{33}+3 \pi_{31}+2\left(\pi_{12}+\pi_{44}\right)+\sqrt{2}\left(2 \pi_{45}-\pi_{16}\right)\right] \sigma d_{\overline{4}} n_{4}^{3} \\
& +\frac{1}{8}\left(S_{11}+S_{33}+2 S_{12}+4 S_{13}-S_{44}-\sqrt{2} S_{16}\right) \sigma d_{\overline{4}}\left(n_{4}-1\right) .
\end{aligned}
$$

Changing pressure direction to $m \perp \bar{B}$ gives rise to a similar relation, which differs by the signs of the terms $\sqrt{2}\left(2 \pi_{45}-\pi_{16}\right)$ and $\sqrt{2} S_{16}$ :

$$
\begin{aligned}
& \delta \Delta_{\overline{4}}=-\frac{1}{16}\left[\pi_{11}+\pi_{13}+\pi_{33}+3 \pi_{31}+2\left(\pi_{12}+\pi_{44}\right)-\sqrt{2}\left(2 \pi_{45}-\pi_{16}\right)\right] \sigma d_{\overline{4}} n_{4}^{3} \\
& +\frac{1}{8}\left(S_{11}+S_{33}+2 S_{12}+4 S_{13}-S_{44}+\sqrt{2} S_{16}\right) \sigma d_{\overline{4}}\left(n_{4}-1\right) .
\end{aligned}
$$

The both formulae are combined in Eq. (T.25), where the upper and the lower signs of the terms $\sqrt{2}\left(2 \pi_{45}-\pi_{16}\right)$ and $\sqrt{2} S_{16}$ correspond to the conditions $m \perp B$ and $m \perp \bar{B}$, respectively.

Following from non-identity of Eqs. (39) and (40) at $m \perp B$ and $m \perp \bar{B}$ (see also Eq. (T.25)), one can conclude that arbitrary choice of the directions $B$ and $\bar{B}$ can lead to ambiguous determination of the POC difference $\sqrt{2}\left(2 \pi_{45}-\pi_{16}\right)$. The same concerns the $\pi_{16}$ coefficient calculated on the basis of Eqs. (37), (38) and (T.23). However, the difference of the two versions of Eq. (T.25) written for the cases of $m \perp B$ and $m \perp \bar{B}$ (see Eq. (T.26)) reveals no ambiguity with respect to the POC combination $\sqrt{2}\left(2 \pi_{45}-\pi_{16}\right)$. Besides, most of the POCs and the $S_{k m}$ coefficients are then excluded from the formula and, as a result, the $2 \pi_{45}-\pi_{16}$ value can be determined with high enough accuracy. The same also refers to Eq. (T.24) used for calculating the POC $\pi_{16}$ (see the comments appearing below Eq. (38)). 
After inserting the $\pi_{16}$ coefficient into Eq. (T.26) and Eq. (T.15) (or Eqs. (T.17)-(T.20)), one can find the POCs $\pi_{45}$ and $\pi_{61}$, respectively. It is obvious that the POC $\pi_{61}$ obtained on the basis of Eqs. (T.17)-(T.20) should reveal a relatively lower error, since the Poisson contribution in these formulae is either absent or associated with s single elastic compliance coefficient $S_{13}$. At the same time, the Poisson strain contribution in Eq. (T.15) is linked with a complicated combination of the coefficients $S_{k m}$, and so the error of each of them will inevitably contribute to the total experimental error.

\subsubsection{A choice of coordinate system}

Let us finally consider another principled question: does the ambiguity of calculation of the POC $\pi_{16}$ and the POC difference $2 \pi_{45}-\pi_{16}$ depend on the choice of right-handed coordinate system? It has been shown in the works $[9,10,14]$ that the ambiguity in the choice of the directions 4 and $\overline{4}$ for the trigonal crystals of the symmetry classes $32,3 \mathrm{~m}$ and $\overline{3} \mathrm{~m}$ does lead to ambiguous calculation of the coefficients $\pi_{14}, \pi_{41}$ and $\pi_{44}$.

In order to solve the problem, let us consider experimental manifestations of the POE for the $B$-cut samples of crystals with the symmetries $4, \overline{4}$ and $4 / \mathrm{m}$. Let us have the two right-handed coordinate systems in which the directions 4 and $\overline{4}$ are interchanged (notice that the directions 4 and $\overline{4}$ in Fig. 8 are interchanged with respect to the corresponding directions presented in Fig. 1e). Then the light polarization can be aligned with the directions $i=1$ and $i=\overline{4}$ (see Fig. 8), the light propagation direction with $k=4$, while the tensors $\left[\sigma_{B}\right]$ and $\left[\sigma_{\bar{B}}\right]$ would differ from those given by Eqs. (35) and (36). Below we will derive the latter tensors. If the condition $\boldsymbol{P} \perp B$ is fulfilled, then the angle between the vector $\boldsymbol{P}$ and the direction 1 is equal to $135^{\circ}$ (see Fig. 8). The directional cosine $a$ of the vector $\boldsymbol{P}$ is equal to $\cos 135^{\circ}=-\sqrt{2} / 2$. It is seen from Fig. 8 that the projection of $\boldsymbol{P}$ on the direction $\overline{4}$ is equal to

$$
P_{\overline{4}}=P \cos 45^{\circ}=P \sqrt{2} / 2
$$

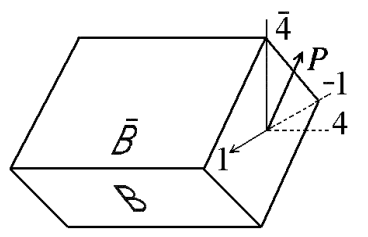

Fig. 8. A scheme of orientations of crystallographic directions 1, 4 and $\overline{4}$ which differ from those shown in Fig. 1e (see the text).

To determine the directional cosines $b$ and $c$, one should find the projections of $\boldsymbol{P}$ upon the axes 2 and 3. The mutual arrangement of the directions $1,2,-2,3,4$ and $\overline{4}$ is schematically represented in Fig. 9. It follows from Fig. 9 that

$$
-P_{2}=P_{\overline{4}} \cos 45^{\circ}=P_{\overline{4}} \sqrt{2} / 2, \quad P_{3}=P_{\overline{4}} \cos 45^{\circ}=P_{\overline{4}} \sqrt{2} / 2 .
$$

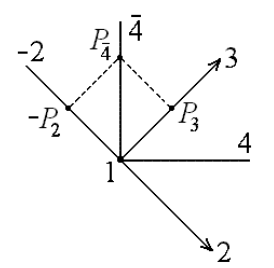

Fig. 9. Arrangement of directions $1,2,-2,3,4$ and $\overline{4}$ for finding projections of the $\boldsymbol{P}$ vector on axes 2 and 3 (see the text).

With accounting for Eq. (41), Eqs. (42) may be rewritten as $-P_{2}=P / 2, P_{3}=P / 2$ or $b=P_{2} / P=-$ $1 / 2, c=P_{3} / P=1 / 2$. Putting the quantity 


$$
P=P(a, b, c)=P(-\sqrt{2} / 2,-1 / 2,1 / 2)
$$

into Eq. (5), one readily obtains the tensor $\left[\sigma_{B}\right]$ :

$$
\left[\sigma_{B}\right]=\sigma\left[\frac{1}{2}, \frac{1}{4}, \frac{1}{4},-\frac{1}{4},-\frac{\sqrt{2}}{4}, \frac{\sqrt{2}}{4}\right] .
$$

Analogically for the case of $\boldsymbol{P} \perp \bar{B}$ we have

$$
P_{\bar{B}}=P_{\bar{B}}(-\sqrt{2} / 2,1 / 2,-1 / 2),
$$

and the tensor $\left[\sigma_{\bar{B}}\right]$ may be presented as

$$
\left[\sigma_{\bar{B}}\right]=\sigma\left[\frac{1}{2}, \frac{1}{4}, \frac{1}{4},-\frac{1}{4}, \frac{\sqrt{2}}{4},-\frac{\sqrt{2}}{4}\right] .
$$

It is seen that the signs of $\sigma_{5}$ and $\sigma_{6}$ have changed, when compared to Eq. (43). Then using the refractive index changes $\delta n_{1}$ and $\delta n_{\overline{4}}$ and the strain $\varepsilon_{4}^{*}$ arising when the stresses defined by Eqs. (43) and (44) are applied, one can calculate the optical path $\delta \Delta_{k}$. For example, at $i=1, k=4$ and $m \perp B$ one gets

$$
\begin{aligned}
\delta \Delta_{4}= & -\frac{1}{8}\left(2 \pi_{11}+\pi_{12}+\pi_{13}+\sqrt{2} \pi_{16}\right) d_{4} \sigma n_{1}^{3} \\
& +\frac{1}{8}\left(S_{11}+S_{33}+2 S_{12}+4 S_{13}-S_{44}-\sqrt{2} S_{16}\right) d_{4} \sigma\left(n_{1}-1\right) .
\end{aligned}
$$

This relation is identical to Eq. (37) used for calculation of the coefficient $\pi_{16}$. Under the condition $m \perp \bar{B}$ one can obtain the relation for $\pi_{16}$ identical to Eq. (38). The same concerns the problem of determination of the POC difference $2 \pi_{45}-\pi_{16}$ : at $i=\overline{4}, k=4$ and $m \perp B$ (or $m \perp \bar{B}$ ) one obtains the relations identical to Eq. (39) or Eq. (40). At the same time, Eqs. (T.24) and (T.26) remain identical in the both coordinate systems (see Fig. 1e and Fig. 8).

Hence, the directions $B$ and $\bar{B}$ are not symmetrically identical for the classes $4, \overline{4}$ and $4 / \mathrm{m}$, unlike the directions 4 and $\overline{4}, 6$ and $\overline{6}$, and $6^{*}$ and $\overline{6}^{*}$. However, the differences of the two versions of Eq. (T.23) and Eq. (T.25) written for the cases of $m \perp B$ and $m \perp \bar{B}$ would result respectively in Eqs. (T.24) and (T.26). The latter relations reveal no ambiguity concerning the problem of calculation of the POCs $\pi_{16}$ and the POC combination $2 \pi_{45}-\pi_{16}$. This conclusion is also true of experimental determination of the POCs $\pi_{45}$ and $\pi_{61}$, the relations for which include the POC $\pi_{16}$. In other words, the right-handed coordinate system for the $B$-cut sample can be chosen arbitrarily, using only the most general recommendations for choosing crystallographic axes for the tetragonal crystals.

\section{Conclusions}

We have derived the analytical relations which describe all of the POCs for the tetragonal crystals belonging to the symmetry classes $4, \overline{4}$ and $4 / \mathrm{m}$. These relations take a micro-wedge shape of real samples into consideration. We have shown that a number experimental geometries associated with indirect cuts of crystal samples enable determining the principal POCs $\pi_{i m}(i, m=1,2,3)$ and their sums of the type $\pi_{11}+\pi_{12}, \pi_{12}+\pi_{13}$, etc. After calculating these POCs on the basis of our theoretical results, one can compare their values $\pi_{i m}$ with those experimentally obtained using the direct-cut samples. Such a comparison seems to be a strong enough test of reliability of the ex- 
perimental results and an effective way to reveal a piezooptic identity of samples obtained from different parts of the same crystalline boule or from different boules.

To determine the POCs for the second subgroup of tetragonal system (i.e., for the point symmetry groups $422,4 \mathrm{~mm}, \overline{4} 2 \mathrm{~m}$, and $4 / \mathrm{mmm}$ ), it is enough to use the analytical relations for the direct-cut, $X / 45^{\circ}$ - and $Z / 45^{\circ}$-cut samples.

\section{References}

1. Narasimhamurty T S, Veerabhadra R K and Pettersen H B, 1973. Photoelastic constants of ADP. J. Mater. Sci. 8: 577-580.

2. Narasimhamurty T S, 1969. Photoelastic behavior of Rochelle salt. Phys. Rev. 186: 945-948.

3. Andrushchak A S, Mytsyk B H, Romashko V A and Seglinsh Ya A, 1991. Piezooptic effect in $\mathrm{Ba}_{\mathrm{x}} \mathrm{Sr}_{1-\mathrm{x}} \mathrm{Nb}_{2} \mathrm{O}_{6}$ crystals. Ukr. Fiz. Zhurn. 36: 618-622.

4. Feldman A, Horowitz D, Waxler R M and Dodge M J. Optical materials characterization. National Bur. Stand. (USA). Tech. Note 993 (1979).

5. Martynyuk-Lototska I, Mys O, Dudok T, Adamiv V, Smirnov Y and Vlokh R, 2008. Acousto-optic interaction in $\alpha-\mathrm{BaB}_{2} \mathrm{O}_{4}$ and $\mathrm{Li}_{2} \mathrm{~B}_{4} \mathrm{O}_{7}$ crystals. Appl. Opt. 47: 3446-3454.

6. Martynyuk-Lototska I, Dudok T, Mys O, Romanyuk G and Vlokh R, 2009. Acoustooptic interaction and photoelastic properties of $\mathrm{Li}_{2} \mathrm{~B}_{4} \mathrm{O}_{7}$ and $\alpha-\mathrm{BaB}_{2} \mathrm{O}_{4}$ crystals at the wavelength of 442 nm. Ukr. J. Phys. Opt. 10: 218-225.

7. Smushkov I V, Kaplan M S and Sumin V I, 1970. The temperature dependence of piezooptic constants of $\mathrm{KCl}$ and KBr. Fiz. Tverd. Tela. 12: 1937-1940.

8. Narasimhamurty T S, Photoelastic and electrooptic properties of crystals. New York: Plenum Press (1981).

9. Mytsyk B H, Andrushchak A S and Gaskevich G I, 2007. Comprehensive studies of piezooptical effect in langasite crystals. Ukr. J. Phys. 52: 800-809.

10. Mytsyk B G, Andrushchak A S, Demyanyshyn N M, Kost YaP, Kityk A V, Mandracci P, Schranz W, 2009. Piezo-optic coefficients of $\mathrm{MgO}$-doped $\mathrm{LiNbO}_{3}$ crystals. Appl. Opt. 48: 1904-1911.

11. Vasylkiv Yu, Savaryn V, Smaga I, Skab I and Vlokh R, 2011. On determination of sign of the piezo-optic coefficients using torsion method. Appl. Opt. 50: 2512-2518.

12. Skab I, Smaga I, Savaryn V, Vasylkiv Yu, Vlokh R, 2011. Torsion method for measuring piezooptic coefficients. Cryst. Res. Tech. 46: 23-36,

13. Sirotin Yu I and Shaskolskaya M P, Fundamentals of crystal physics. Moscow: Nauka (1979).

14. Mytsyk B, 2003. Methods for the studies of the piezo-optical effect in crystals and the analysis of experimental data. Part I. Methodology for the studies of piezo-optical effect. Ukr. J. Phys. Opt. 4: $1-26$.

Mytsyk B., Demyanyshyn N. and Kost' Ya., 2013. Analytical relations describing piezooptic effect in tetragonal crystals. Ukr.J.Phys.Opt. 14: $101-118$.

Анотація. Одержано аналітичні співвідношення для визначення усіх n'єзооптичних коефіиієнтів за інтерферометричним методом із урахуванням мікроклиновидності реальних зразків. Представлено результати для тетрагональних класів симетрії 4, $\overline{4}$ i 4/m. Співвідношення для точкових груп 422, 4mm, $\overline{4} 2 m$ i 4/mmm є простими частковими випадками загальних співвідношень для n'єзооптичного ефекту в тетрагональних кристалах. 